\title{
Perfusion CT in suspected ischaemic stroke: red flags that should have been blue
}

\author{
Mark H. B. Huisman • Birgitta K. Velthuis • \\ L. Jaap Kappelle $\cdot$ Geert Jan Biessels
}

Received: 19 May 2010/Revised: 21 July 2010/Accepted: 21 July 2010/Published online: 12 August 2010

(C) The Author(s) 2010. This article is published with open access at Springerlink.com

Dear Sirs,

In patients suspected of stroke it is important to make the correct diagnosis as soon as possible because thrombolysis might be indicated. We describe two cases with an acute neurological deficit suggestive of stroke. In both cases, noncontrast $\mathrm{CT}$ scans were compatible with cerebral ischaemia. However, perfusion CT showed hyperperfusion and the diagnosis cerebral ischaemia was rejected. These cases illustrate the value of perfusion CT in the diagnostic process of suspected ischaemia.

Acute focal neurological deficit of cerebral origin is usually caused by ischaemic or hemorrhagic stroke. Less frequent other causes are hypoglycaemia, epilepsy, migraine, multiple sclerosis, intracranial neoplasms or somatoform disorders [1]. It is important to diagnose cerebral ischaemia as soon as possible because thrombolysis might be indicated. Perfusion CT might be of help in making a correct diagnosis in the hyperacute stage. The typical features of ischaemia on perfusion $\mathrm{CT}$ are decreased cerebral blood flow (CBF) and a prolonged mean transit time (MTT). Cerebral blood volume (CBV) may also be decreased, indicating irreversibility of the ischaemia. In most centres these perfusion abnormalities are reflected by

M. H. B. Huisman $(\bowtie) \cdot$ L. J. Kappelle · G. J. Biessels Department of Neurology, G03.228, University Medical Centre Utrecht, Rudolf Magnus Institute of Neurosciences, P.O. Box 85500, 3508 GA Utrecht, The Netherlands e-mail: m.h.b.huisman-3@umcutrecht.nl

B. K. Velthuis

Department of Radiology, University Medical Centre Utrecht, P.O. Box 85500, 3508 GA Utrecht, The Netherlands a blue colour on the colour-coded perfusion maps [2]. We describe two patients with acute neurological deficit in whom perfusion CT made it possible to make a correct diagnosis in the hyperacute stage.

An 84-year-old man with atrial fibrillation, hypertension and dyslipidemia presented with acute expressive aphasia and mild right-sided hemiparesis that existed for two and a half hours. He used warfarin because of atrial fibrillation, which was still present on the electrocardiogram. The international normalised ratio was 2.32 (optimal value: 3.00). Cerebral ischaemia was suspected.

Noncontrast brain CT showed a left parietal hypodensity (Fig. 1d). Perfusion CT showed abnormalities in the same region, compatible with hyper- rather than hypoperfusion (Fig. 1a-c). MRI demonstrated a lesion, most likely being a high-grade glioma (Fig. 1e, f). The patient declined further investigations and he died 1 month later.

A 71-year-old woman with diabetes mellitus type 2 presented with sudden dysarthria, hemianopsia and left-sided hemiparesis and neglect. Nine days earlier she had undergone right carotid endarterectomy because of a symptomatic stenosis greater than $70 \%$. Blood pressure was $180 / 80 \mathrm{~mm} \mathrm{Hg}$ and she had a regular pulse of 80 beats per minute. During examination, a generalised tonic-clonic seizure occurred. Noncontrast CT showed no new abnormalities as compared to MRI 2 months before. On CT angiography, the right internal carotid artery was patent (Fig. 2a, b). Perfusion CT showed asymmetry between the right and left hemispheres, with increased flow on the right (Fig. 2d-f). Cerebral hyperperfusion syndrome (CHS) was diagnosed and she was treated with antihypertensives.

One day later, MRI showed subcortical hyperintensities on the fluid attenuated inversion recovery (FLAIR) and T2-weighted sequence, but no hyperintensity on the 

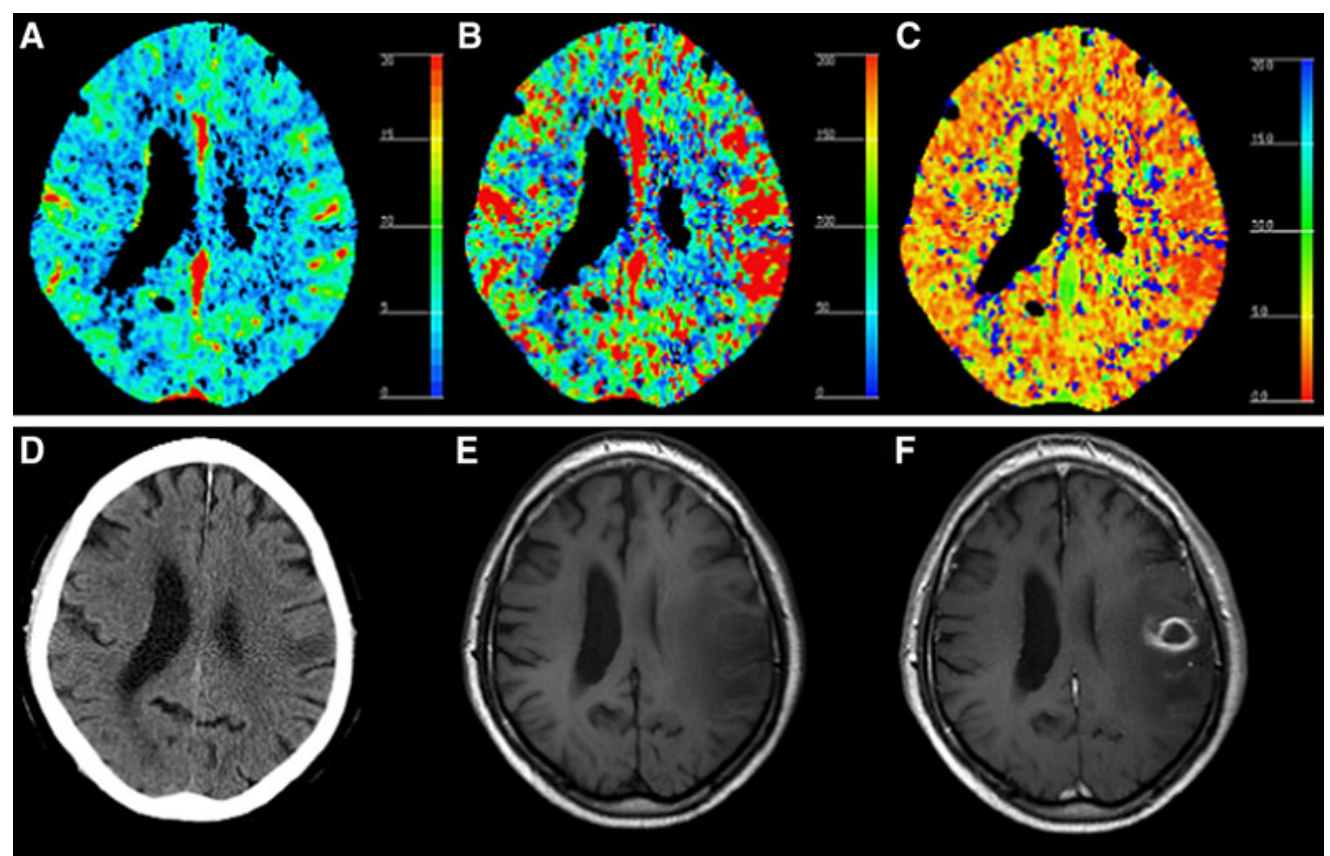

Fig. $1 \mathrm{CT}$ and MRI findings of patient 1. CT perfusion demonstrates an elevated cerebral blood volume (CBV) (a) and cerebral blood flow (CBF) (b) and a decreased mean transit time (MTT) (c) in the left parietal area, compatible with increased perfusion. Noncontrast CT reveals a left parietal hypodensity (d). T1-weighted MRI shows a left parietal hypointense lesion (e) with irregular rim enhancement after gadolinium injection (f) diffusion-weighted sequence or corresponding hypointensity on the apparent diffusion coefficient (ADC) map (Fig. $2 g-1$ ). This ruled out new ischaemia and was compatible with CHS.

These two cases illustrate that perfusion CT can help to identify causes other than cerebral ischaemia in patients with acute neurological deficit.

The first case presented with symptoms compatible with acute cerebral ischaemia in the context of multiple vascular risk factors. Noncontrast CT showed an area of hypodensity, which can be an early sign of ischaemic stroke [3], although in this case it was already relatively pronounced in relation to symptom onset. In our patient, perfusion CT, with increased rather than decreased perfusion, suggested another cause. An intracranial neoplasm is diagnosed in $0.4 \%$ of patients with a transient neurological deficit [4]. Based on the clinical presentation it is not always possible to differentiate between transient attacks that are caused by a tumour or by a primarily vascular cause [5]. Neuroimaging is therefore necessary in patients with sudden focal neurological deficit of cerebral origin. However, it may still be impossible to differentiate cerebral ischaemia from a cerebral tumour on a noncontrast scan.
In the second case, perfusion CT helped to differentiate CHS from ischaemia. Although the recent carotid endarterectomy and the seizure were suggestive for CHS [6], a firm distinction between CHS and ischaemia could not be made solely on the clinical findings and the noncontrast CT. Making this distinction had substantial consequences for treatment.

The two cases illustrate the value of perfusion CT in the diagnostic process of suspected ischaemia. Perfusion CT can be obtained with most multidetector CT scanners with only a few minutes of additional scan-time [7]. Previous studies have established the accuracy of perfusion CT to detect ischaemic stroke [8]. Less is known about its capacity to differentiate between cerebral ischaemia and nonvascular causes of neurological deficits with a sudden onset. Case series now report perfusion abnormalities in stroke mimics, such as hyperperfusion in the context of seizures [9, 10]. Our two cases demonstrate that perfusion $\mathrm{CT}$ is also able to differentiate a tumour or CHS that presents with neurological deficits with a sudden onset from cerebral ischaemia in the acute stage. In both cases, perfusion CT demonstrated increased (red) rather than decreased (blue) flow. In other words, red flags that should have been blue. 

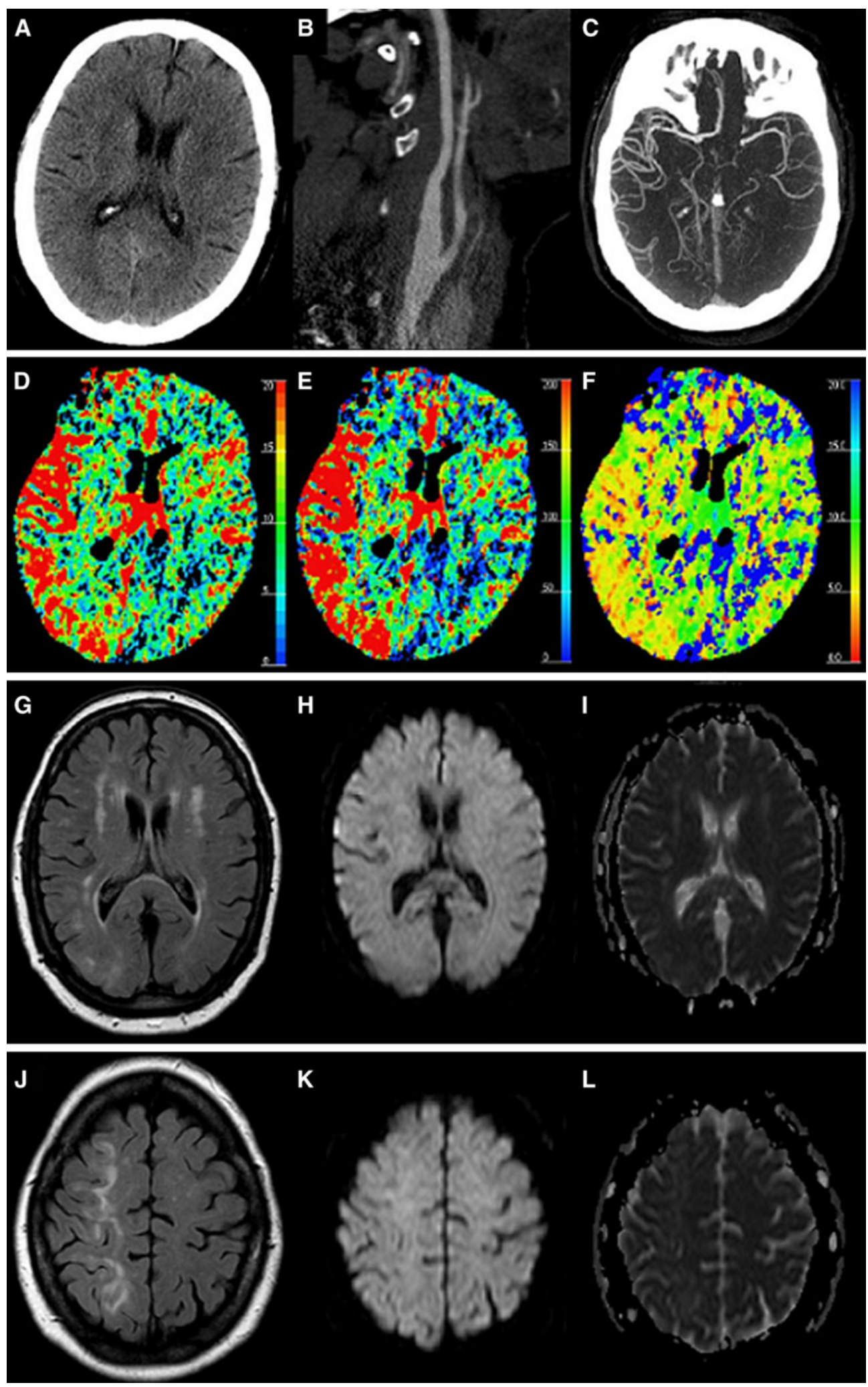

Fig. $2 \mathrm{CT}$ and MRI findings of patient 2. Noncontrast CT shows periventricular white matter lesions (a). CT angiography shows a patent right carotid artery (b) and pronounced intracerebral arteries in the right hemisphere (c) Perfusion CT demonstrates an elevated CBV (d) and CBF (e) and a decreased MTT in the right hemisphere (f).
MRI 1 day later shows, in comparison to a recent previous MRI (not shown), unchanged periventricular white matter lesions (g) and new hyperintensities in the subcortical white matter on the FLAIR sequence (j) and no hyperintensities on the diffusion-weighted sequence $(\mathbf{h}, \mathbf{k})$ or hypointensities on the $\operatorname{ADC}$ map $(\mathbf{i}, \mathbf{l})$ 
Conflicts of interest There are no conflicts of interest/disclosures to report.

Open Access This article is distributed under the terms of the Creative Commons Attribution Noncommercial License which permits any noncommercial use, distribution, and reproduction in any medium, provided the original author(s) and source are credited.

\section{References}

1. Hand PJ, Kwan J, Lindley RI, Dennis MS, Wardlaw JM (2006) Distinguishing between stroke and mimic at the bedside: the brain attack study. Stroke 37:769-775. doi:10.1161/01.STR. $0000204041.13466 .4 \mathrm{c}$

2. Tomandl BF, Klotz E, Handschu R, Stemper B, Reinhardt F, Huk WJ, Eberhardt KE, Fateh-Moghadam S (2003) Comprehensive imaging of ischemic stroke with multisection CT. Radiographics 23:565-592. doi:10.1148/rg.233025036

3. von Kummer R, Bourquain H, Bastianello S, Bozzao L, Manelfe C, Meier D, Hacke W (2001) Early prediction of irreversible brain damage after ischemic stroke at CT. Radiology 219:95-100

4. The UK TIA Study Group (1993) Intracranial tumours that mimic transient cerebral ischaemia: lessons from a large multicentre trial. J Neurol Neurosurg Psychiatr 56:563-566. doi: 10.1136/jnnp.56.5.563

5. García-Moncó JC, Marrodán A, Foncea Beti N, Gómez Beldarrain $M$ (2002) Stroke and transient ischemic attack-mimicking conditions: a prospective analysis of risk factors and clinical profiles at a general hospital. Neurologia 17:355-360

6. van Mook WN, Rennenberg RJ, Schurink GW, van Oostenbrugge RJ, Mess WH, Hofman PA, de Leeuw PW (2005) Cerebral hyperperfusion syndrome. Lancet Neurol 4:877-888. doi:10.1016/ S1474-4422(05)70251-9

7. Parsons MW (2008) Perfusion CT: is it clinically useful? Int J Stroke 3:41-50

8. Wintermark M, Flanders AE, Velthuis B, Meuli R, van Leeuwen M, Goldsher D, Pineda C, Serena J, van der Schaaf I, Waaijer A, Anderson J, Nesbit G, Gabriely I, Medina V, Quiles A, Pohlman S, Quist M, Schnyder P, Bogousslavsky J, Dillon WP, Pedraza S (2006) Perfusion-CT assessment of infarct core and penumbra: receiver operating characteristic curve analysis in 130 patients suspected of acute hemispheric stroke. Stroke 37:979-985. doi: 10.1161/01.STR.0000209238.61459.39

9. Royter V, Paletz L, Waters MF (2008) Stroke vs. status epilepticus. A case report utilizing CT perfusion. J Neurol Sci 266:174-176. doi:10.1016/j.jns.2007.08.038

10. Masterson K, Vargas MI, Delavelle J (2009) Postictal deficit mimicking stroke: role of perfusion CT. J Neuroradiol 36:48-51. doi:10.1016/j.jns.2007.08.038 